\title{
Notch1 is overexpressed in human intrahepatic cholangiocarcinoma and is associated with its proliferation, invasiveness and sensitivity to 5-fluorouracil in vitro
}

\author{
WEN-RUI WU ${ }^{1}$, RUI ZHANG ${ }^{1}$, XIANG-DE SHI ${ }^{1}$, MAN-SHENG ZHU ${ }^{1}$, \\ LEI-BO XU ${ }^{1}$, HONG ZENG ${ }^{2}$ and CHAO LIU ${ }^{1}$ \\ Departments of ${ }^{1}$ Hepato-Pancreato-Biliary Surgery and ${ }^{2}$ Pathology, Sun Yat-sen Memorial \\ Hospital, Sun Yat-sen University, Guangzhou, Guangdong 510120, P.R. China \\ Received January 27, 2014; Accepted February 24, 2014
}

DOI: 10.3892/or.2014.3123

\begin{abstract}
The Notch signaling pathway has been reported to play crucial roles in inhibiting hepatocyte differentiation and allowing formation of intrahepatic bile ducts. However, little is known about its significance in intrahepatic cholangiocarcinoma (ICC). The aim of the present study was to investigate the effects of Notch1 expression in ICC tissues and cells. The expression of Notch1 was examined in paraffin-embedded sections of ICC $(n=44)$ by immunohistochemistry. Notch1 was knocked down by RNA interference (RNAi) in cultured ICC cells (RBE and HCCC-9810). The proliferation, invasiveness and sensitivity to 5-fluorouracil (5-FU) were detected by Cell Counting Kit-8 (CCK-8), colony formation assays, Transwell assays and flow cytometry, respectively. The expression levels of several multidrug resistance (MDR)-related genes, MDR1-P-glycoprotein (ABCB-1), breast cancer resistance protein (ABCG-2) and the multidrug resistance protein isoform 1 (MRP-1), were examined by quantitative real-time polymerase chain reaction (qRT-PCR) and western blotting. Notch1 was overexpressed in cell membranes and cytoplasm of ICC compared with the adjacent liver tissue (35/44, 79.5\%) and this was more common in cases with tumor size $\geq 5 \mathrm{~cm}$ $(\mathrm{p}=0.021)$ and HBs-Ag positive $(\mathrm{p}=0.018)$. By silencing Notch1, the proliferation and invasiveness of ICC cells were inhibited and the inhibition rate of 5-FU was markedly increased. In addition, $\mathrm{IC}_{50}$ values of 5-FU in RBE cells were decreased from $148.74 \pm 0.72$ to $5.37 \pm 0.28 \mu \mathrm{g} / \mathrm{ml}$ and the corresponding values for HCCC-9810 cells were $326.92 \pm 0.87$ to $42.60 \pm 0.35 \mu \mathrm{g} / \mathrm{ml}$, respectively. Furthermore, Notch1 silencing clearly increased
\end{abstract}

Correspondence to: Dr Chao Liu, Department of HepatoPancreato-Biliary Surgery, Sun Yat-sen Memorial Hospital, Sun Yat-sen University, 107 Yan Jian Xi Lu, Guangzhou, Guangdong 510120, P.R. China

E-mail: mdliuchao@hotmail.com

Key words: Notch1, intrahepatic cholangiocarcinoma, RNA interference, chemotherapy the percentage of apoptotic cells treated by 5-FU compared with the control. Notch1 knockdown led to diminished expression levels of ABCB-1 and MRP-1. Therefore, Notch may play important roles in the development of ICC. Silencing Notch1 can inhibit the proliferation and invasiveness of ICC cells and increase their sensitivity to 5-FU in vitro.

\section{Introduction}

Intrahepatic cholangiocarcinoma (ICC) is a life-threatening and treatment-refractory disease with a dismal outcome. The lethality of the disease is due to both rapid tumor growth and the tendency to invade adjacent organs and metastasize (1). Although ICC is relatively rare, its incidence in the Western world is showing an alarming rise (2). ICC accounts for $13 \%$ of all annual cancer-related deaths worldwide and for $3 \%$ of deaths in Western countries (3). To date, little is known about the mechanism of tumor initiation, progression, metastasis formation and drug resistance of ICC. Due to advances in surgical techniques and peri-operative management, the best curative treatment for this malignancy is complete resection with histologically negative tumor margins (4). However, only $13-27 \%$ of patients are eligible for surgical treatment due to extensive perineural invasion, lymphatic metastasis and vascular encasement (5). For the majority of patients presenting with advanced disease, systemic chemotherapy has been disappointing, as ICC has low sensitivity to most chemotherapeutic drugs, including 5-fluorouracil (5-FU) (6,7).

Notch is an evolutionarily-conserved single-pass transmembrane receptor involved in numerous cell fate decisions during development, stem cell renewal and differentiation in postnatal tissues. Accumulating evidence suggests that Notch signaling plays a critical role in the development of several types of cancer, functioning as a tumor promoter or a tumor suppressor (8-15).

In the present study, we reported common upregulation of Notch1 in ICC tissues (35/44, 79.5\%). In functional analysis, we showed knockdown of Notch1 could reduce cell proliferation and invasiveness of ICC cells. Also, depletion of Notch1 sensitized ICC cells to the chemotherapeutic drug 5-FU. The identification and functional characterization of Notch 1 in 
ICC provides new insights into the possible use of Notch1 as a therapeutic target.

\section{Materials and methods}

Patients and tumor tissue specimens. Forty-four surgicallyresected liver tissue specimens of ICC were examined. None of the patients had received chemotherapy or radiation therapy prior to the radical tumor resection. The project was approved by the Ethics Committee of the Hospital and was in accordance with the Helsinki Declaration of 1975. Written informed consent was obtained from either the patients or their guardians.

Immunohistochemical staining. Immunohistochemical staining was carried out according to the protocol defined in the PV Two-Step kit instructions (Zhongshan Goldenbridge Biotechnology, Beijing, China) . Briefly, sections of a paraffinembedded tissue block were deparaffinized twice in xylene for $15 \mathrm{~min}$ and rehydrated through graded ethanol solutions. Sections were subsequently heated in a microwave oven twice for antigen retrieval for $8 \mathrm{~min}$. Citrate buffer $(10 \mathrm{mmol} / \mathrm{l}$, $\mathrm{pH}$ 6.0) was used as the antigen retrieval buffer. Endogenous peroxidases were inactivated by immersing the sections in $0.3 \%$ hydrogen peroxide for $15 \mathrm{~min}$. Then, the slides were incubated at $4^{\circ} \mathrm{C}$ overnight in a humidified chamber with Notch1 monoclonal antibody (Cell Signaling Technology Inc., Boston, MA, USA) in a final dilution of 1:100. The sections were further incubated with goat anti-rabbit immunoglobulin G-horseradish peroxidase conjugate for $30 \mathrm{~min}$ at room temperature. Finally, the sections were developed with DAB color solution (50 $\mu \mathrm{l} / \mathrm{section})$ for $2 \mathrm{~min}$ at room temperature. Hematoxylin (Boster Biotechnology, Wuhan, China) was then used as a chromogen (50 $\mu \mathrm{l} /$ section) and the slides were consecutively counter-stained for $30 \mathrm{sec}$. With the exception of the omission of primary antibodies, negative controls were processed in the same manner as above. All sections were washed three times in phosphate-buffered saline (PBS; pH 7.4) for $5 \mathrm{~min}$ after each step.

Semi-quantitative method for the immunohistochemical expression of Notch1. The semi-quantitative method was applied for the immunohistochemical expression of Notch1. The percentages of positively stained cells were determined by examination under a microscope of 5 randomly selected foci, composed of $>100$ cells each. Scoring was based on distribution and intensity according to a previous report (16).

Cell culture. Two human ICC cell lines, RBE and HCCC-9810, were purchased from the Chinese Academy of Sciences Shanghai Branch Cell Bank and cultured in RPMI-1640 (Gibco BRL, Gaithersburg, MD, USA) supplemented with $10 \%$ heatinactivated fetal bovine serum (FBS; Biological Industries, Kibbutz Beit Haemek, Israel) as recommended by the supplier. All cultures were maintained in a humidified atmosphere containing $5 \% \mathrm{CO}_{2}$ at $37^{\circ} \mathrm{C}$.

siRNA interference. For the RNAi analyses, human Notch1 small interfering RNA (siRNA) with the nucleotide sequence 5'-UACAGUACUGACCUGUCCACUCUGG-3' (sense) and 5'-CCAGAGUGGACAGGUCAGUACUGUA-3' (antisense), corresponding to part of the Notch1 mRNA were designed and purchased from Shanghai GenePharma (Shanghai, China). As a negative control, we used a control scrambled siRNA oligonucleotide (NC siRNA; sense, 5'-UUCUCCGAACGUGUCACG UTT-3', antisense, 5'-ACGUGACACGUUCGGAGAATT-3') targeting a sequence not sharing homology with the human genome (GenePharma). Briefly, RBE and HCCC-9810 cells were cultured in RPMI-1640 medium containing 10\% FBS, incubated for $18-22 \mathrm{~h}$ at $37^{\circ} \mathrm{C}, 5 \% \mathrm{CO}_{2}$ and grown to $50 \%$ confluence in well plates. Cells were transfected using the Lipofectamine $^{\mathrm{TM}} 2000$ reagent (Invitrogen, Carlsbad, CA, USA) according to the manufacturer's protocol. Notch1 siRNA or NC siRNA (final $100 \mathrm{nM}$ ) solutions were prepared and the siRNA-Lipofectamine transfection complexes were incubated for $20 \mathrm{~min}$ at room temperature. The culture medium was replaced with OPTI-MEM (Invitrogen) and suspension was added drop-wise onto the cells. After $6 \mathrm{~h}$ of transfection, the culture medium was recovered to a normal medium (RPMI-1640 medium containing 10\% FBS).

RNA isolation and real-time $q R T-P C R$ analysis. Total RNA was isolated using RNAiso Plus reagent according to the manufacturer's protocol (Takara Biotechnology, Tokyo, Japan). cDNA was synthesized using the PrimeScript RT Reagent (Takara). Portions of double-stranded cDNA were subjected to PCR with a SYBR-Green Premix Ex Taq (Takara). Primer sets (design and synthesis by Takara) used for qRT-PCR are shown in Table I. As a control, the levels of glyceraldehyde phosphate dehydrogenase (GAPDH) expression were also analyzed. The amplification protocol comprised incubations at $95^{\circ} \mathrm{C}$ for $30 \mathrm{sec}, 95^{\circ} \mathrm{C}$ for $5 \mathrm{sec}$ and $65^{\circ} \mathrm{C}$ for $20 \mathrm{sec}$. Incorporation of the SYBR-Green dye into PCR products was monitored in realtime with LightCycler real-time PCR detection system (Roche Applied Science, Indianapolis, IN, USA), thereby allowing for the determination of the threshold cycle $(\mathrm{Ct})$ at which exponential amplification of products begins. Fold change was determined by $2^{-\Delta \Delta \mathrm{Ct}}$.

Protein purification and western blot analysis. Cells were rinsed three times with cold PBS and lysed on ice with a lysis buffer [50 mM Tris (pH 7.4), $150 \mathrm{mM} \mathrm{NaCl,} \mathrm{1 \%} \mathrm{NP-40,}$ $0.1 \% \mathrm{SDS}$ ], $1 \%$ protease inhibitor phenylmethane-sulfonyl fluoride (PMSF; Bocai Bio, Shanghai, China) for $30 \mathrm{~min}$ and centrifuged down to collect the supernatant. Protein concentrations in the supernatant were determined using a BCA protein assay kit (Bocai Bio). Proteins were separated on $10 \%$ SDS-PAGE gels and transferred to a PVDF membrane followed by western blot analysis. In brief, $5 \%$ milk in TBS containing $0.1 \%$ Tween-20 was used to block the non-specific binding. The blot was subsequently incubated with primary antibodies for Notch1 (1:1,000; Cell Signaling Technology), MRP-1 (1:50, Chemicon Inc., Temecula, CA, USA), ABCB-1 (1:1,000; Epitomics Inc., Burlingame, CA, USA), ABCG-2 (1:1,000; Epitomics) and with the goat anti-rabbit secondary antibody (1:2,000; Cell Signaling Technology). $\beta$-tubulin (1:2,000; Cell Signaling Technology) was used as an internal control. After each antibody incubation, blots were extensively washed in TBS containing $0.1 \%$ Tween-20. For detection, we used the ECL Plus Western Blotting Detection System (Amersham Biosciences, Piscataway, NJ, USA). The intensity 
Table I. Primers for Notch1, MRP-1, ABCB-1, ABCG-2 and reference genes.

\begin{tabular}{ll} 
Gene & \multicolumn{1}{c}{ Primer Sequence } \\
\hline Notch1 & F: 5'-GAGGCGTGGCAGACTATGC-3' \\
& R: 5'-CTTGTACTCCGTCAGCGTGA-3 \\
MRP-1 & F: 5'-GAGGAACCATATTACAGGTCCGT-3' \\
& R: 5'-AGGGGATCATCGAAGAGGTAAAT-3' \\
ABCB-1 & F: 5'-GGGAGCTTAACACCCGACTTA-3' \\
& R: 5'-GCAAAATCACAAGGGTTAGCTT-3' \\
ABCG-2 & F: 5'-CAGGTGGAGGCAAATCTTCGT-3' \\
& R: 5'-ACCCTGTTAATCCGTTCGTTTT-3' \\
GAPDH & F: 5'-ACAACTTTGGTATCGTGGAAG-3' \\
& R: 5'-ACAACTTTGGTATCGTGGAAG-3'
\end{tabular}

F, forward; R, reverse.

of western bands was measured by the Quantity One software (Bio-Rad Laboratories Inc., Hercules, CA, USA).

Cell proliferation assay by $C C K-8$. One day before transfection, the cells $\left(5 \times 10^{3} /\right.$ well $)$ were cultured in 96-well tissue culture plates and then transfected with Notch1 siRNA or NC siRNA. After transfection $(24,48$ and $72 \mathrm{~h}$ ), viability of the cells was determined using the Cell Counting Kit-8 (CCK-8) that was purchased from the Dojindo Molecular Technologies (Dojindo China Co., Shanghai, China). Briefly, after washing in PBS, $100 \mu \mathrm{l}$ medium containing 10\% CCK-8 solution were added to each well and incubated for $2 \mathrm{~h}$ at $37^{\circ} \mathrm{C}$. Samples were read directly in the wells using an absorbance of the $450 \mathrm{~nm}$ wavelength by an enzyme linked immunosorbent assay (ELISA) plate reader. In order to adjust the background, the blank control absorbance (100 $\mu \mathrm{l}$ medium containing $10 \%$ CCK-8 alone) was subtracted from the experimental absorbance.

Colony formation assay. Twenty-four hours after transfection, the transfected cells were seeded in 6-well tissue culture plates ( 500 cells/well). After an incubation period of 10 days, the medium was decanted and each well was washed twice with PBS. The cells were stained with $1 \%$ crystal violet (in $100 \%$ methanol) for $15 \mathrm{~min}$, followed by detaining. Colonies (>20 cells/colony) were counted.

Transwell invasive assay. Twenty-four hours after transfection, $1 \times 10^{5}$ cells in $0.1 \mathrm{ml}$ serum-free medium were placed into the upper chamber (24-well plate) of the insert (Corning Inc., Corning, NY, USA) with Matrigel (BD Biosciences, San Jose, CA, USA), whereas the lower chamber was $0.6 \mathrm{ml}$ RPMI-1640 medium containing 20\% FBS. After $24 \mathrm{~h}$ of incubation, the cells that were still on the upper side of the filters were mechanically removed with cotton swabs. Cells that had migrated to the lower side were fixed with $4 \%$ paraformaldehyde and were counterstained with $0.1 \%$ crystal violet. The cells that had migrated into the lower chamber were observed and counted under a light microscope. Finally, the number of the migrating cells was calculated.

Measurement of cytotoxicity by $C C K-8$. Twenty-four hours after transfection, the cells were treated with various concentrations $(50,100,200$ and $400 \mu \mathrm{g} / \mathrm{ml}$, respectively) of 5-FU (Baiyunshan Pharmaceutical Co., Guangzhou, China) for $48 \mathrm{~h}$. Then, the cell viability was determined by CCK- 8 assay. The rate of cell growth inhibition (IR) was calculated according to the following equation: IR $=[1-\mathrm{A} 450(\mathrm{drug}) / \mathrm{A} 450$ (control)] $\mathrm{x} 100 \%$, where A450 (drug) is the absorbance of the cells exposed to 5-FU and A450 (control) is the absorbance of the cells without 5-FU treatment.

DAPI staining. 4,6-Diamidino-2-phenylindole (DAPI; Invitrogen) staining was performed according to the manufacturer's protocol. In brief, cells were fixed with $4 \%$ paraformaldehyde for $30 \mathrm{~min}$, washed three times with cold PBS, then exposed to $1 \mu \mathrm{g} / \mathrm{ml}$ DAPI solution for $15 \mathrm{~min}$ in the dark at room temperature. Stained cells were observed under a laser scanning microscope (Nikon, Tokyo, Japan).

Flow cytometry. Twenty-four hours after transfection, cells were treated with 5 -FU (50 and $100 \mu \mathrm{g} / \mathrm{ml}$ ) for $48 \mathrm{~h}$. To detect the apoptosis of ICC cells, the cells were doubly stained with Annexin V-FITC (BD Bioscience) and propidium iodide (Sigma Chemical Co., St. Louis, MO, USA) followed by flow cytometry analysis. Apoptotic ratio was determined on the basis of Annexin $\mathrm{V}^{+} \mathrm{PI}^{+}$and Annexin $\mathrm{V}^{+} \mathrm{PI}-$ fractions.

Statistical analysis. Each assay was performed in triplicate. Statistical analysis was conducted with the SPSS software package (version 13.0; SPSS, Inc., Chicago, IL, USA). Data are presented as the means \pm standard deviation (SD). The relation between the expression of Notch1 in ICC samples and the clinico-pathological data was statistically analyzed by $\chi^{2}$ test, and the other data was compared using paired Student's t-test. P-values $<0.05$ were considered to indicate statistically significant differences.

\section{Results}

Notch1 is overexpressed in ICC. Notch1 was overexpressed in cell membranes and cytoplasm of human ICC compared with the adjacent liver tissue (35/44, 79.5\%; Fig. 1). Among them, Notch1 was more common in cases with tumor size $>5 \mathrm{~cm}$ $(95.5 \%, 21 / 22)$ than in cases with tumor size $\leq 5 \mathrm{~cm}(63.6 \%$, $14 / 22, p=0.021$ ). In addition, Notch 1 was more expressed in ICC patients with HBs-Ag positive $(100 \%, 15 / 15)$ than in patients with HBs-Ag negative $(69.0 \%, 20 / 29, p=0.018)$. There was no statistically significant difference in age, gender, liver cirrhosis, capsular invasion, portal vein tumor thrombi, bile duct tumor thrombi, lymphatic or organ metastasis, tumor number, tumor stage, histological grade, serum $\alpha$ fetoprotein (AFP) level, serum CA199 level and serum CA125 level between Notch1 positive and Notch1 negative patients (Table II).

siRNAs targeting Notchl gene downregulate Notch expression in ICC cells. To address the functional importance of the Notch1 gene, we employed RNAi to deplete its expression in RBE and 
A

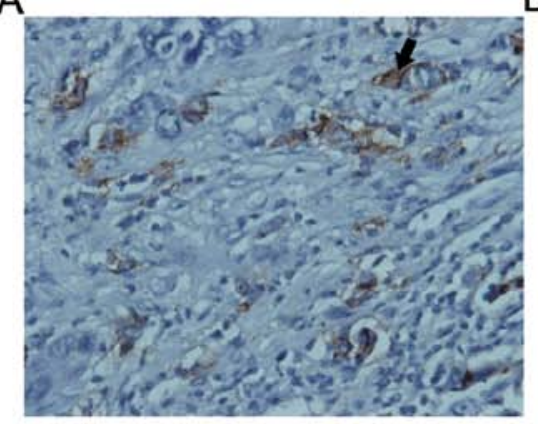

B

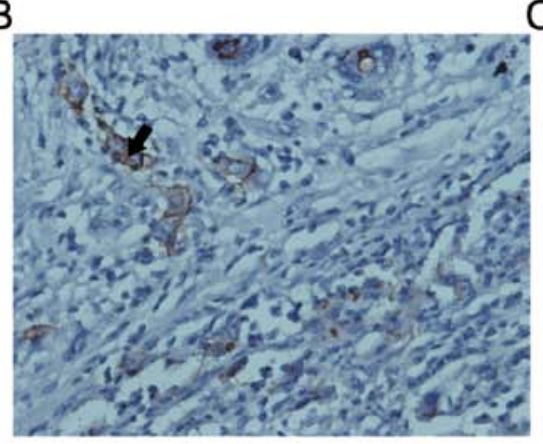

C

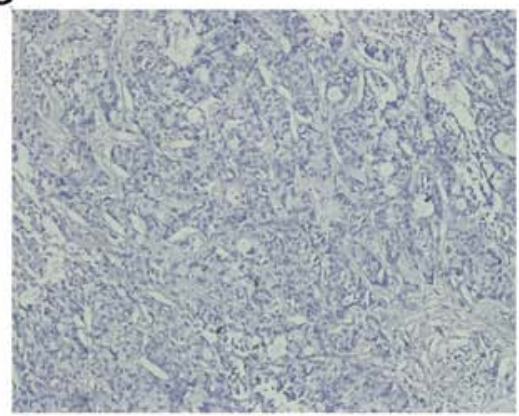

Figure 1. Overexpression of Notch1 in ICC. Brown-colored expression of Notch1 (black arrows) is located at cell membranes and cytoplasm. (A) Original magnification, x200. (B) Original magnification, x400. Negative controls were obtained by omitting the primary antibody. (C) Original magnification, x100.
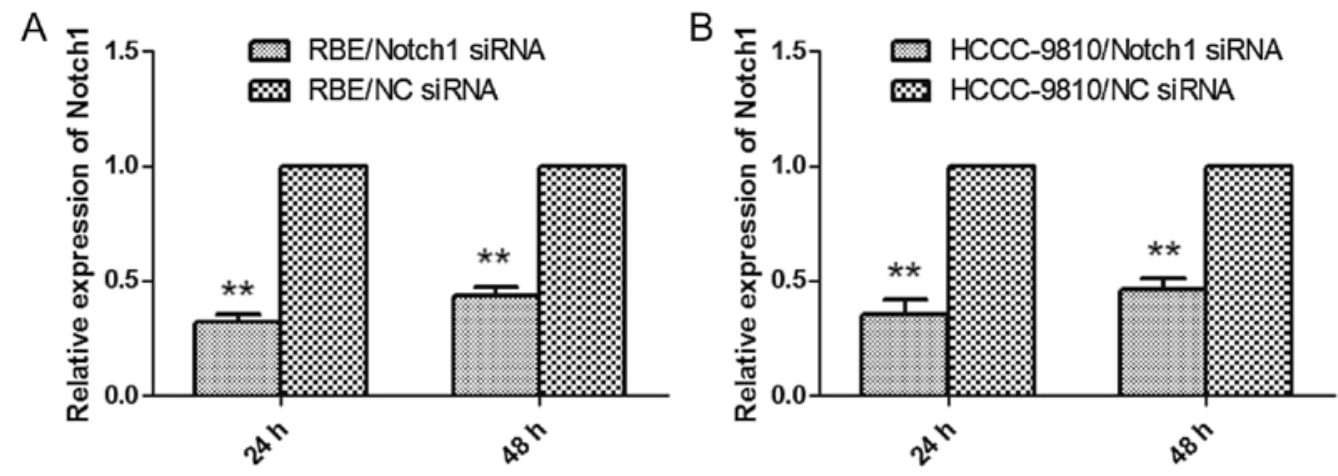

Figure 2. Downregulation of Notch1 expression in ICC cells by RNAi. qRT-PCR analysis showed that the mRNA expression levels of Notch1 in (A) RBE cells and (B) HCCC-9810 cells were significantly downregulated. ${ }^{* *} \mathrm{P}<0.01$.
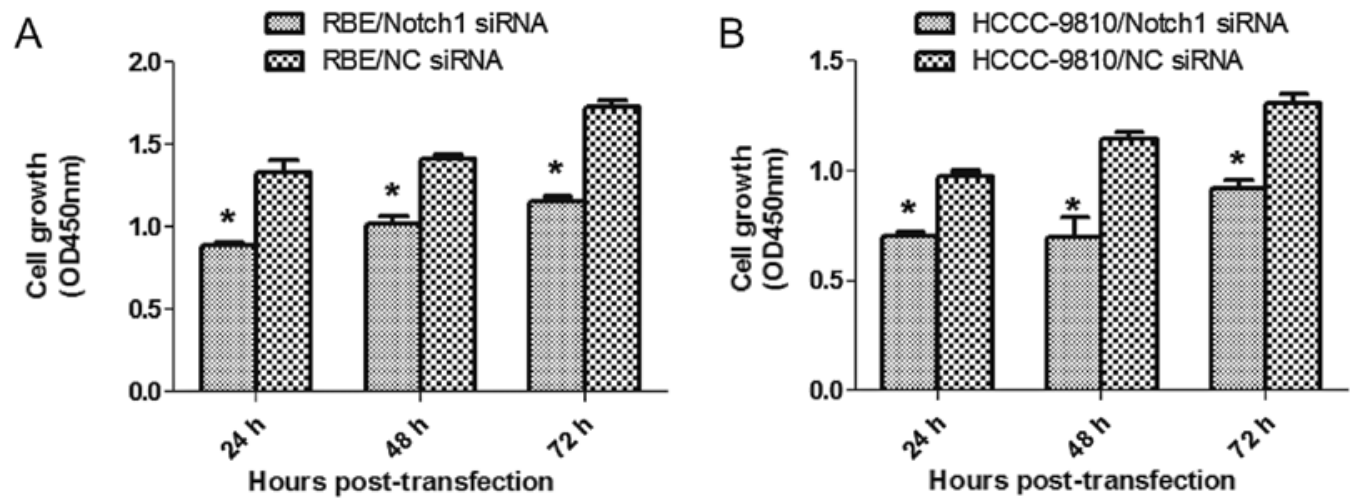

Figure 3. Knockdown of Notch1 suppresses in vitro proliferation of ICC cells. Downregulation of Notch1 expression by Notch1-siRNA significantly inhibited the growth of (A) RBE and (B) HCCC-9810 cells. ${ }^{*} \mathrm{P}<0.05$.

HCCC-9810 cells, both of which were treated with NC-siRNA or siRNA targeting Notch1. After $24 \mathrm{~h}$, the cells were examined by qRT-PCR and western blot analysis. As shown in Fig. 2A and B, the gene expression was markedly knocked down as determined by qRT-PCR. Similar results were observed in the western blot analysis (Fig. 9C). These data indicated that Notch1-specific siRNA clearly and effectively suppressed the expression of Notch1 in RBE and HCCC-9810 cells.

Depletion of Notch1 suppresses ICC cell proliferation. To this end, CCK-8 assay was performed 24, 48 and $72 \mathrm{~h}$ after transfection. Compared to the NC-siRNA, Notch1-siRNA transfection inhibited the growth of RBE and HCCC-9810 cells in vitro $(\mathrm{P}<0.05$; Fig. $3 \mathrm{~A}$ and $\mathrm{B})$. Also, fewer numbers were formed in Notch1-depletion cells from colony formation assay ( $\mathrm{P}<0.001$; Fig. 4). Our results suggested that depletion of Notch1 inhibited proliferation and growth of ICC.

Notch1-siRNA diminishes the invasive ability of ICC cells. To examine whether targeted downregulation of Notchl in RBE and HCCC-9810 cells affects the invasive ability of tumor cells, in vitro Transwell invasive assays were performed. The number of tumor cells migrating through the filter in the Notch1-siRNA group was markedly lower than that in 
Table II. Clinicopathological data of 44 intrahepatic cholangiocarcinoma patients.

\begin{tabular}{|c|c|c|c|c|}
\hline \multirow[b]{2}{*}{ Characteristics } & \multirow[b]{2}{*}{$\mathrm{N}$} & \multicolumn{2}{|c|}{ Group } & \multirow[b]{2}{*}{ P-value } \\
\hline & & Notch1+ & Notch1- & \\
\hline Age (years) & & & & 0.659 \\
\hline$\leq 50$ & 8 & 6 & 2 & \\
\hline$>50$ & 36 & 29 & 7 & \\
\hline Gender & & & & 0.227 \\
\hline Male & 32 & 27 & 5 & \\
\hline Female & 12 & 8 & 4 & \\
\hline Cirrhosis & & & & 0.566 \\
\hline Yes & 4 & 4 & 0 & \\
\hline No & 40 & 31 & 9 & \\
\hline Capsular invasion & & & & 0.124 \\
\hline Yes & 16 & 15 & 1 & \\
\hline No & 28 & 20 & 8 & \\
\hline Portal vein tumor thrombi & & & & 1.000 \\
\hline Yes & 12 & 10 & 2 & \\
\hline No & 32 & 25 & 7 & \\
\hline Bile duct tumor thrombi & & & & 1.000 \\
\hline Yes & 3 & 3 & 0 & \\
\hline No & 41 & 32 & 9 & \\
\hline Lymphatic metastasis & & & & 0.477 \\
\hline Yes & 24 & 18 & 6 & \\
\hline No & 20 & 17 & 3 & \\
\hline Organ invasion & & & & 0.716 \\
\hline Yes & 17 & 13 & 4 & \\
\hline No & 27 & 22 & 5 & \\
\hline Tumor number & & & & 0.319 \\
\hline Single & 38 & 29 & 9 & \\
\hline Multiple & 6 & 6 & 0 & \\
\hline Tumor size & & & & 0.021 \\
\hline$\leq 5 \mathrm{~cm}$ & 22 & 14 & 8 & \\
\hline$>5 \mathrm{~cm}$ & 22 & 21 & 1 & \\
\hline Tumor stage (UICC, 2010) & & & & 0.411 \\
\hline I + II & 11 & 10 & 1 & \\
\hline III + IV & 33 & 25 & 8 & \\
\hline Histological grade & & & & 0.235 \\
\hline $\mathrm{G} 1+\mathrm{G} 2$ & 29 & 25 & 4 & \\
\hline $\mathrm{G} 3+\mathrm{G} 4$ & 15 & 10 & 5 & \\
\hline HBs-Ag & & & & 0.018 \\
\hline Positive & 15 & 15 & 0 & \\
\hline Negative & 29 & 20 & 9 & \\
\hline Serum AFP & & & & 0.566 \\
\hline$\leq 25 \mathrm{ng} / \mathrm{ml}$ & 40 & 31 & 9 & \\
\hline$>25 \mathrm{ng} / \mathrm{ml}$ & 4 & 4 & 0 & \\
\hline CA199 & & & & 0.092 \\
\hline$>35 \mu / \mathrm{ml}$ & 34 & 25 & 9 & \\
\hline$\leq 35 \mu / \mathrm{ml}$ & 10 & 10 & 0 & \\
\hline CA125 & & & & 0.262 \\
\hline$>35 \mu / \mathrm{ml}$ & 20 & 18 & 2 & \\
\hline$\leq 35 \mu / \mathrm{ml}$ & 26 & 19 & 7 & \\
\hline
\end{tabular}



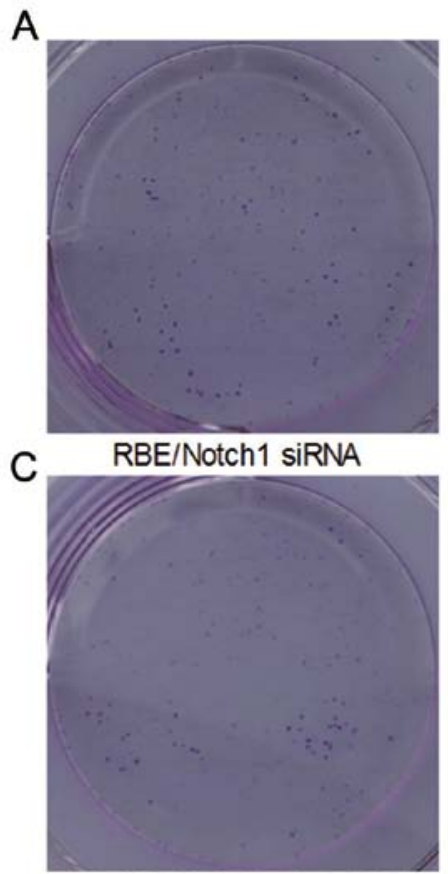

HCCC-9810/Notch1 siRNA

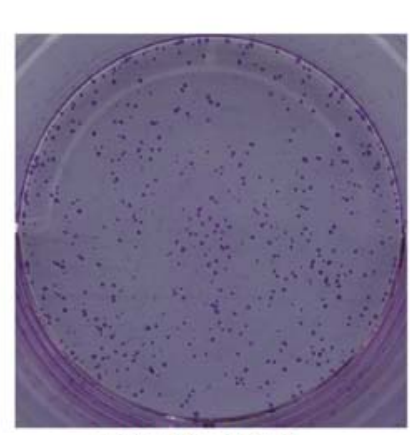

RBE/NC SiRNA

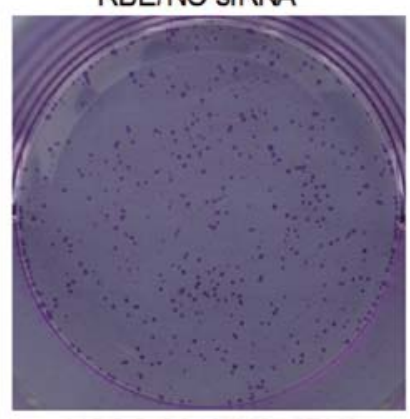

HCCC-9810/NC siRNA
B

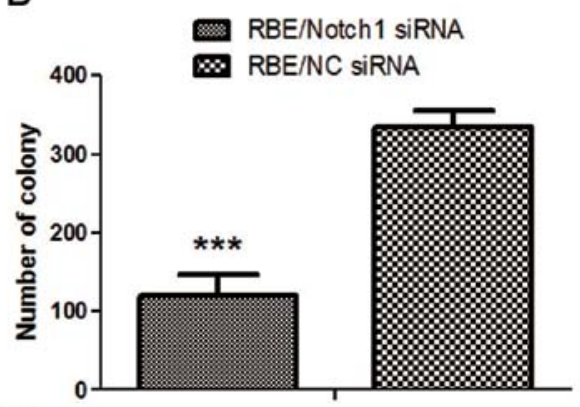

D

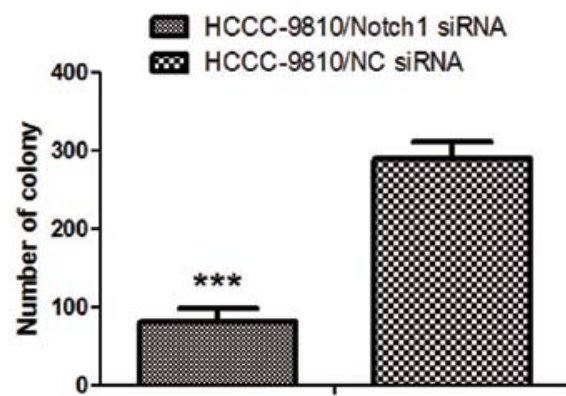

Figure 4. Knockdown of Notch1 suppresses colony formation of ICC cells. Colony formation assays showed the reduction of colony formation ability of (A and B) RBE and (C and D) HCCC-9810 cells after knockdown of Notch1. ${ }^{* * *} \mathrm{P}<0.001$.

A

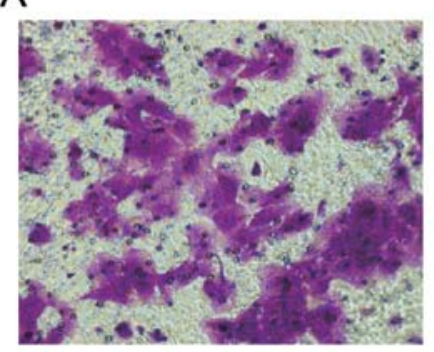

RBE/Notch1 siRNA

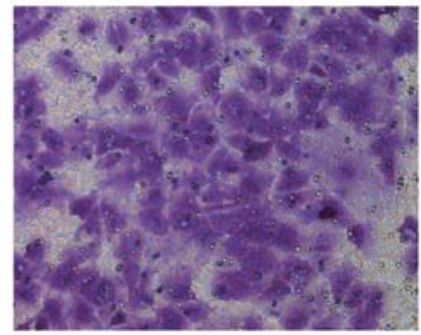

RBE/NC siRNA

\section{B}

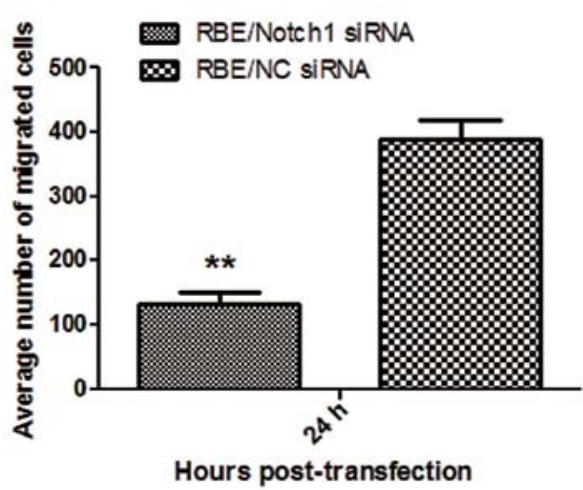

C
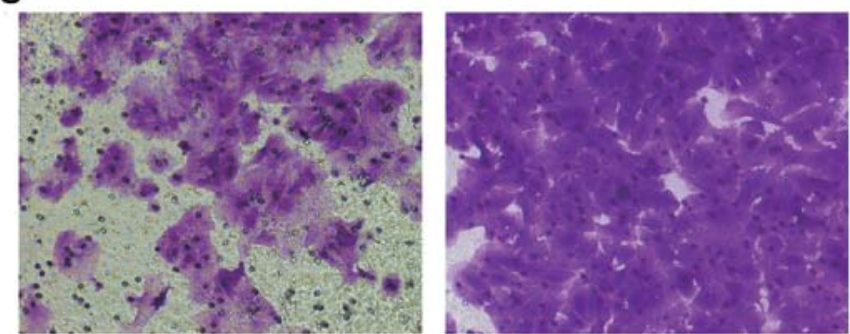

HCCC-9810/Notch1 siRNA HCCC-9810/Notch1 siRNA

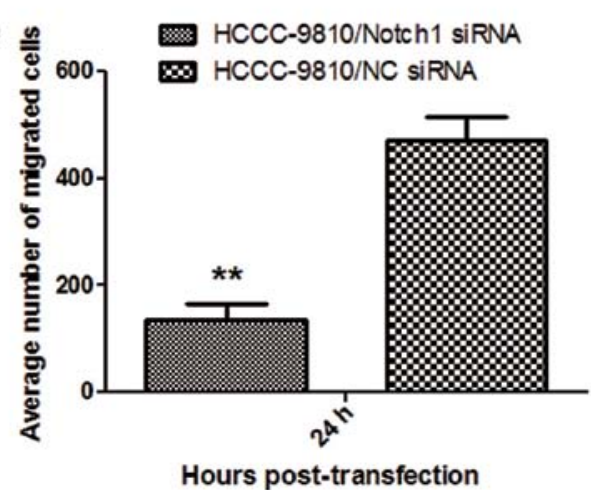

Figure 5. Depletion of Notch1 inhibits invasiveness of ICC cells. The effect of Notch1 knockdown on the invasiveness of RBE and HCCC-9810 cells. The number of (A and B) RBE and (C and D) HCCC-9810 cells that migrated through the filters was markedly lower in the Notch1-siRNA group than in the NC-siRNA group. ${ }^{* *} \mathrm{P}<0.01$.

the NC-siRNA group (P<0.01; Fig. 5 ). Thus, Notch1-siRNA silencing markedly diminished the invasiveness of RBE and HCCC-9810 cells in vitro.
Knockdown of Notchl sensitizes ICC cells to 5-FU-induced apoptosis. To further explore the role of Notch1 in ICC, we tested whether downregulation of Notch1 by RNAi sensitizes 
A

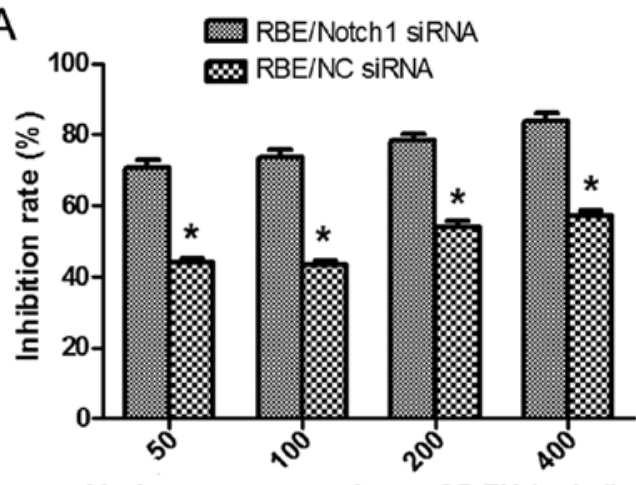

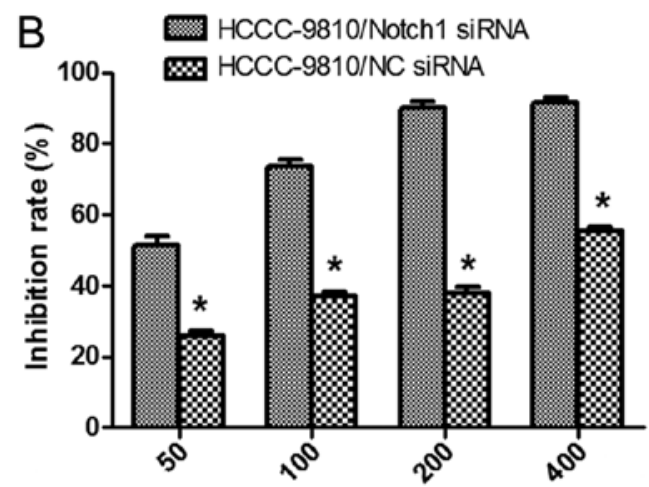

Various concentrations of $5-\mathrm{FU}(\boldsymbol{\mu g} / \mathrm{ml})$

Figure 6. Depletion of Notch1 sensitizes ICC cells to 5-FU treatment. The inhibition rate of 5-FU in the Notch1-siRNA transfected ICC cells (A) RBE and (B) HCCC-9810 was significantly increased compared to the control. ${ }^{*} \mathrm{P}<0.05$.
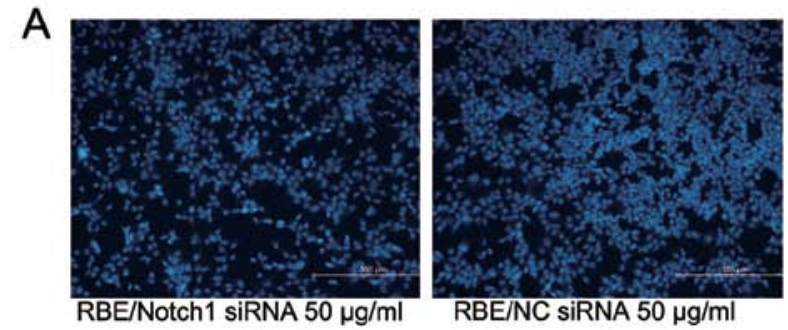

B

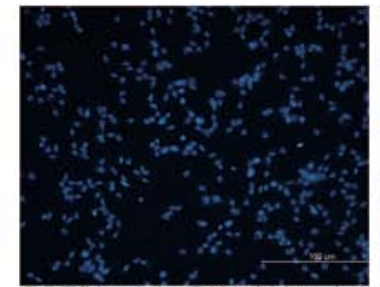

B
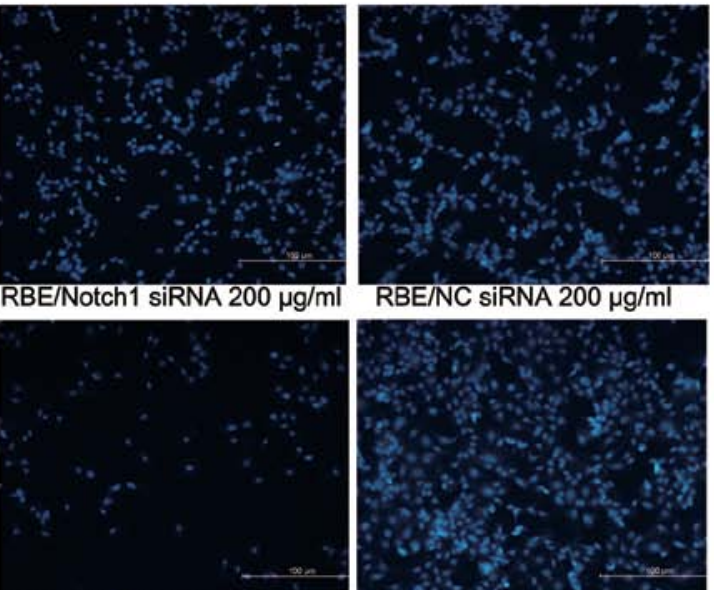

HCCC-9810/Notch1 siRNA $50 \mu \mathrm{g} / \mathrm{ml}$ HCCC-9810/NC SiRNA $50 \mu \mathrm{g} / \mathrm{m}$
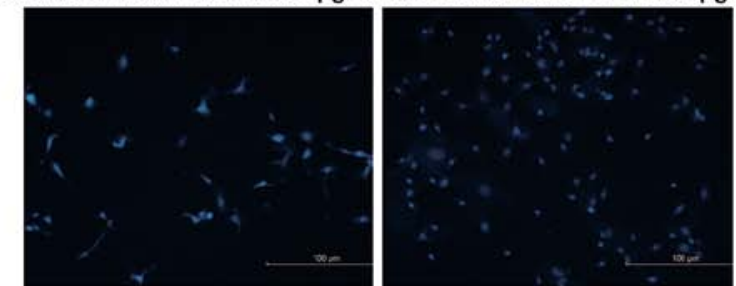
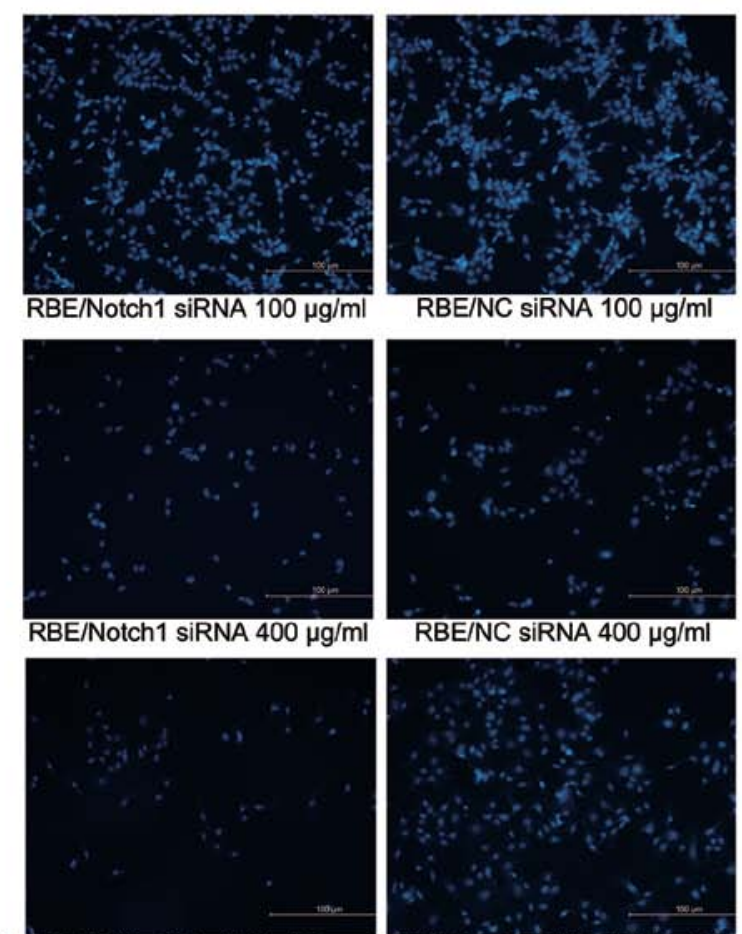

RBE/NC siRNA $100 \mu \mathrm{g} / \mathrm{ml}$
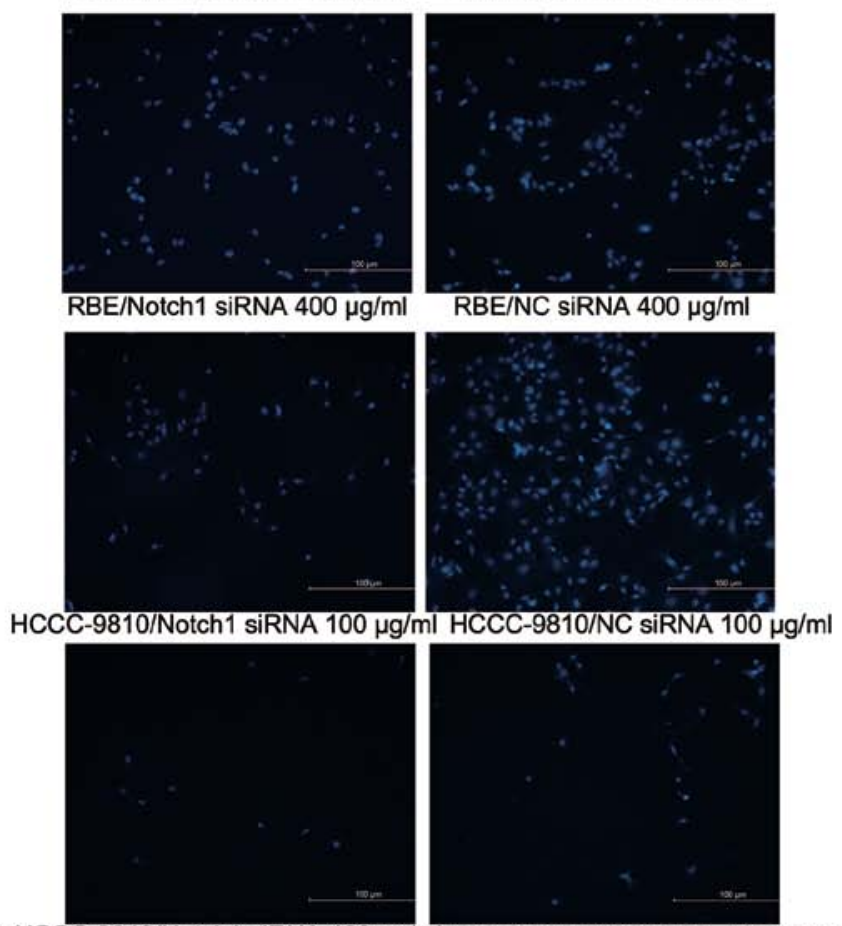

HCCC-9810/Notch 1 siRNA $200 \mu \mathrm{g} / \mathrm{ml}$ HCCC-9810/NC siRNA $200 \mu \mathrm{g} / \mathrm{ml}$ HCCC-9810/Notch1 siRNA $400 \mathrm{\mu g} / \mathrm{ml}$ HCCC-9810/NC siRNA $400 \mu \mathrm{g} / \mathrm{ml}$

Figure 7. Effect of Notch1 downregulation on 5-FU-treated ICC cells. Fluorescence images (magnification, x100) of (A) RBE cells and (B) HCCC-9810 cells stained with DAPI.

ICC cells to 5-FU chemotherapy. After transfection and treatment with various concentrations of 5-FU, cell viability was determined by CCK- 8 assay. The results showed that both the Notch1-siRNA transfected cells showed lower cell viability than the control $(\mathrm{P}<0.05$; Fig. 6$)$. The $\mathrm{IC}_{50}$ values of 5-FU in the RBE-NC-siRNA and RBE-Notch1-siRNA cells were $148.74 \pm 0.72 \mu \mathrm{g} / \mathrm{ml}$ and $5.37 \pm 0.28 \mu \mathrm{g} / \mathrm{ml}$, respectively, and the corresponding values for HCCC-9810-NC-siRNA and HCCC-9810-Notch1-siRNA cells were $326.92 \pm 0.87$ and $42.60 \pm 0.35 \mu \mathrm{g} / \mathrm{ml}$, respectively. In order to confirm these results, the cells were subjected to DAPI staining. Both RBE-Notch1-siRNA and HCCC-9810-Notch1-siRNA cells showed lower cell numbers than the control cells (Fig. 7A and B). We also examined the apoptotic rate of 


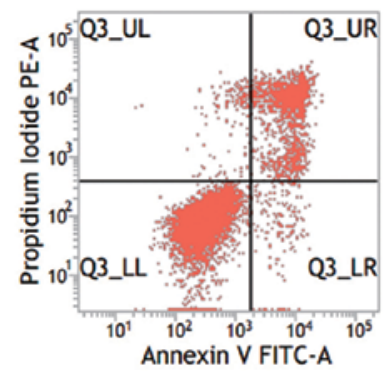

RBE/Notch 1 siRNA $50 \mu \mathrm{g} / \mathrm{ml}$ apoptotic rate: $(18.22 \pm 1.74) \%$

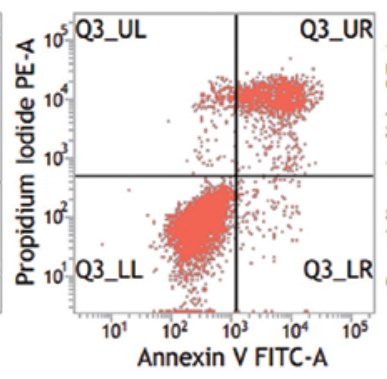

RBE/NC siRNA $50 \mu \mathrm{g} / \mathrm{ml}$ apoptotic rate: $(14.67 \pm 1.21) \%$

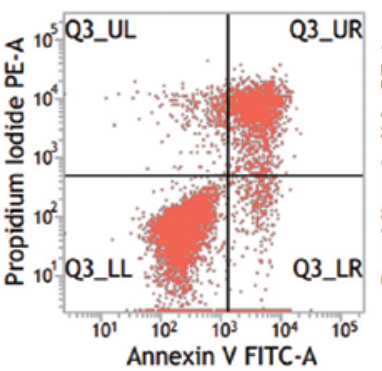

RBE/Notch1 siRNA $100 \mu \mathrm{g} / \mathrm{ml}$ apoptotic rate: $(28.00 \pm 1.86) \%$

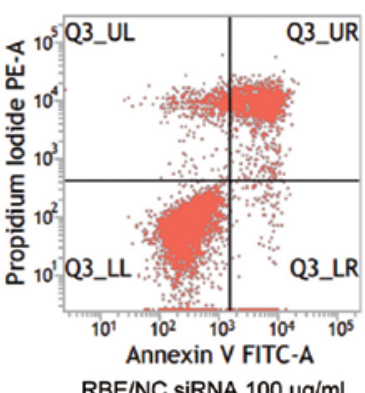

RBE/NC SiRNA $100 \mu \mathrm{g} / \mathrm{ml}$ apoptotic rate: $(19.64 \pm 1.91) \%$

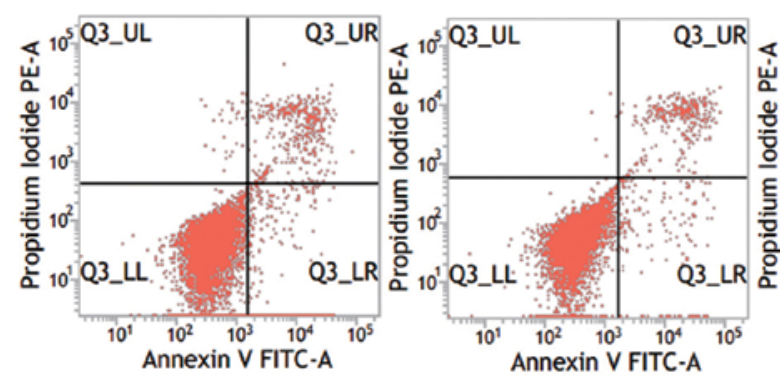

HCCC-9810/Notch 1 siRNA $50 \mu \mathrm{g} / \mathrm{ml}$ HCCC-9810/NC siRNA $50 \mu \mathrm{g} / \mathrm{ml}$ apoptotic rate: $(10.52 \pm 1.52) \%$

apoptotic rate: $(5.21 \pm 1.88) \%$
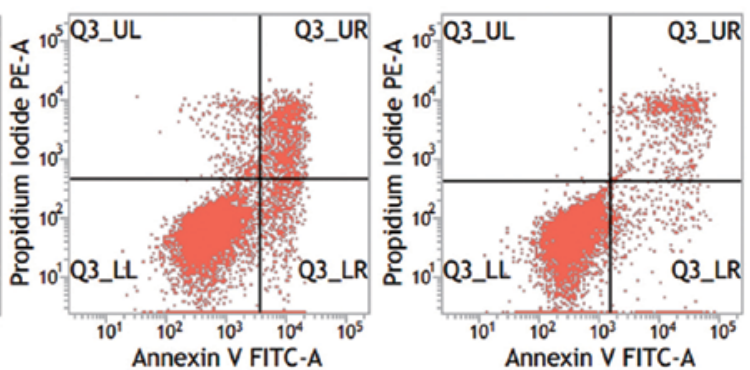

HCCC-9810/Notch1 siRNA $100 \mu \mathrm{g} / \mathrm{ml}$ HCCC-9810/NC siRNA $100 \mu \mathrm{g} / \mathrm{ml}$ apoptotic rate: $(16.01 \pm 1.47) \%$ apoptotic rate: $(10.79 \pm 1.32) \%$

Figure 8. Notch1 downregulation increases apoptotic rates of ICC cells treated by 5-FU. Flow cytometry analyses of propidium iodide-stained cells to 5-FU and the percentages of apoptotic cells.
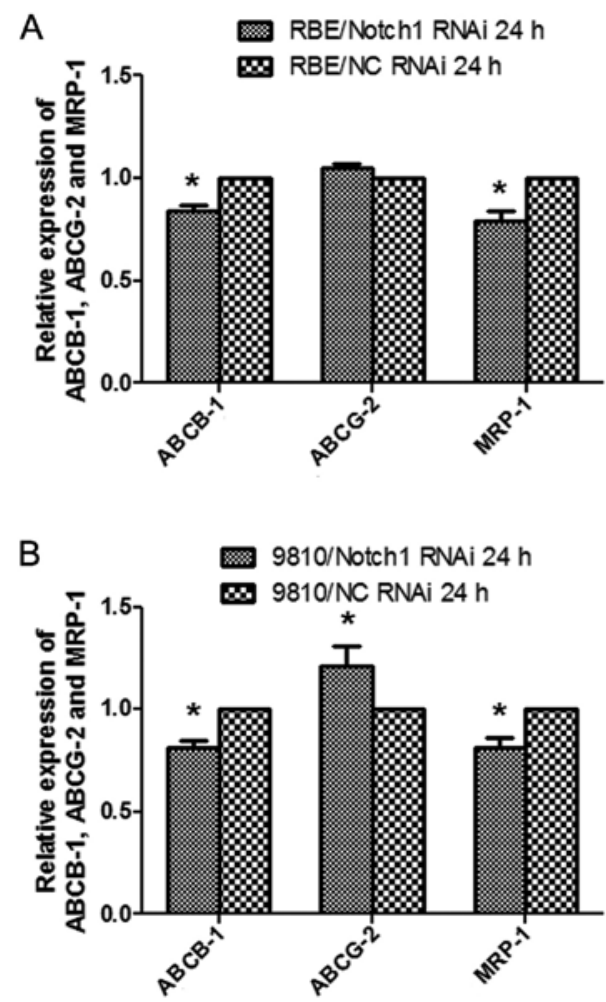

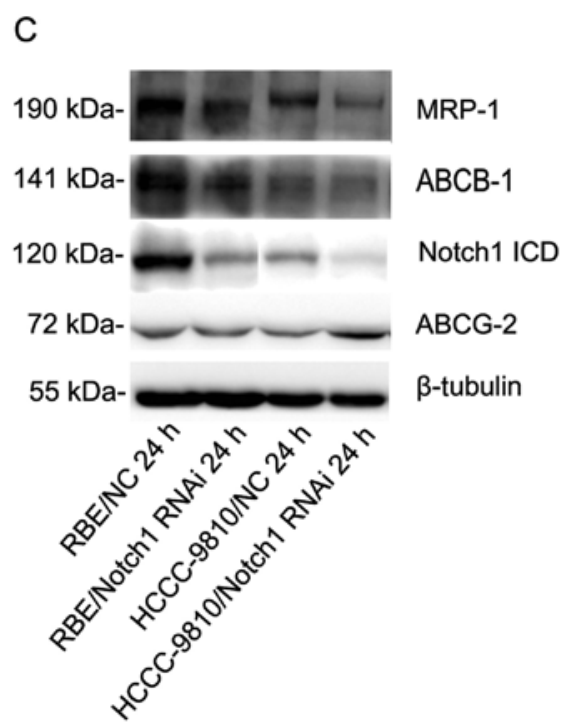

Figure 9. Notch1 influences the expression of ABCB-1 and MRP-1 in ICC. Knockdown of Notch1 diminished the expression of ABCB-1 and MRP-1 at both (A and B) mRNA and (C) protein levels. ${ }^{*} \mathrm{P}<0.05$.

tumor cells by flow cytometry. The results showed that the apoptotic rates of the RBE-Notch1-siRNA and HCCC-9810Notch1-siRNA cells were much higher than those of the
RBE-NC-siRNA and HCCC-9810-NC-siRNA cells, respectively $(\mathrm{P}<0.01$; Fig. 8). These results indicated that knockdown of Notch1 sensitized ICC cells to 5-FU treatment. 
Knockdown of Notchl diminishes the expression of ABCB-1 and MRP-1. To further explore the mechanism underlying the suppression of ICC cell resistance to 5-FU treatment by the silencing of Notch1, the expression levels of the MDR-related genes, ABCB-1, ABCG-2 and MRP-1, were examined $24 \mathrm{~h}$ after transfection by qRT-PCR and western blotting. The results showed that knockdown of Notch1 diminished the expression of ABCB-1 and MRP-1 in both cell lines $(\mathrm{P}<0.05$; Fig. 9). Silencing Notch1 in RBE cells did not affect the expression of ABCG-2. However, ABCG-2 was increased in HCCC-9810-Notch1-siRNA cells but not in the control.

\section{Discussion}

A majority of ICC patients who undergo chemotherapy show MDR, which is often responsible for therapy failure and poor outcome. To identify signaling pathways that could be targeted to enhance ICC sensitivity to drug-based therapies is of outmost importance. However, the precise molecular mechanisms of MDR remain obscure. One explanation for MDR is overexpression of membrane transport proteins such as ABCB-1, ABCG-2 and MRP-1, which act as efflux pumps for anticancer agents (17). In addition, resistance to apoptosis also contributes to chemoresistance (18).

The Notch signaling pathway plays a critical role in cell fate decision, tissue patterning, morphogenesis and is hence regarded as a developmental pathway. If it goes awry, it would lead to cellular transformation and tumorigenesis (19). It is generally accepted that the Notch signaling pathway is deregulated in a variety of malignancies and can behave as either an oncogene or a tumor suppressor gene depending on cell type. In the study of liver cancer, Wang et al reported that Notch1/Jagged1 were frequently low expressed in hepatocellular carcinoma and downregulation of Notch1/Jagged1 may sustain tumor progression (20). It has also been demonstrated that Notch1 signaling inhibits growth of human hepatocellular carcinoma through induction of cell cycle arrest and apoptosis. However, in extrahepatic cholangiocarcinoma and gallbladder carcinoma, Notch1 was overexpressed and correlated with cancer progression (16). Furthermore, regarding the relationship between Notch and HBV infection, Trehanpati et al found that HBV infection drives increased Notch1, TGF- $\beta$ and FoxP3 expression on intrahepatic T-cells in cirrhosis, resulting in fibrogenesis and disease progression (21). Pei et al confirmed that blockage of Notch1 signaling could regulate the immune balance of Th1/Th2 in chronic hepatitis B patients (22). Moreover, there is mounting evidence that Notch1 plays an important role in the process of MDR. Nefedova $e t$ al verified that Notch-1 signaling maybe a primary mechanism mediating the bone marrow stroma influence on hematologic malignant cell growth and survival from drug-induced apoptosis (23). Zou et al affirmed that in hypoxic conditions Notch1 signaling is required to activate genes regulating cellular proliferation, invasion and chemoresistance, increasing the aggressiveness of T-ALL and its likelihood for progression (24).

In the present study, immunohistochemical analysis showed that Notch1 was overexpressed in the cell membrane and cytoplasm of human ICC $(35 / 44,79.5 \%)$ compared with the adjacent liver tissue and was more common in cases with tumor size $\geq 5 \mathrm{~cm}$ and $\mathrm{HBs}-\mathrm{Ag}$ positive, suggesting that their overexpression may be linked to cancer initiation (hepatitis B virus infection) and progression. To examine the role of Notch1 expression in ICC, cell culture studies were performed. The Notch1 gene was silenced by Notch1-siRNA in 2 ICC cell lines, RBE and HCCC-9810. Then, the proliferation and invasiveness of these cells were detected by CCK-8, colony formation and Transwell assays. The results showed that the proliferation and invasion ability of tumor cells in the Notch1-silenced group were significantly lower than those in the control group. Loss of Notch1 in ICC specifically resulted in their enhanced 5-FU mediated death as revealed by multiple criteria: (1) Annexin V-FITC/PI staining, (2) CCK-8 analysis, (3) DAPI staining. We observed that Notch1 gene silencing significantly increased the apoptotic rate of tumor cells treated by 5-FU and significantly decreased the $\mathrm{IC}_{50}$ values of 5-FU. These results showed that knockdown of endogenous Notch1 expression of ICC cells inhibited their proliferation and invasiveness, contributed to sensitization of ICC cells to 5-FU and increased their apoptotic rates, which suggest that the combination of conventional chemotherapy and Notch1-gene target therapy may be a potential clinical strategy for ICC therapy. To further investigate the mechanism of how Notch1 ablation enhances apoptosis in response to 5-FU treatment, we examined ABCB-1, ABCG-2 and MRP-1 levels in the presence and absence of Notch1 by qRT-PCR and western blot analysis. The results showed that knockdown of endogenous Notch1 expression led to significantly downregulated ABCB-1 and MRP-1 expression levels at both mRNA and protein levels in these two cell lines; this indicated that Notch1-RNAi enhanced apoptosis in response to 5-FU treatment cells, which may partly be mediated through MDR-related genes. Since the expression of ABCG-2 was beyond our expectation, there may be complicated regulatory mechanisms of Notch1 and MDR-related genes that remain undefined. This requires further elucidation. In conclusion, we have shown in the present study that Notch1 is upregulated in ICC and promotes tumor proliferation, invasiveness and chemoresistance in vitro, indicating that Notch1 may be involved in ICC carcinogenesis and progression. These findings suggest that Notch1 could serve as a novel therapeutic target in patients with ICC and further investigations on the signaling network of Notch1 may provide new insight into this fatal disease.

\section{Acknowledgements}

This study was supported by the Special Research Foundation of the National Natural Science Foundation of China (81172068).

\section{References}

1. Guglielmi A, Ruzzenente A and Campagnaro T, et al: Intrahepatic cholangiocarcinoma: prognostic factors after surgical resection. World J Surg 33: 1247-1254, 2009.

2. Goodman ZD: Neoplasms of the liver. Mod Pathol 20 (Suppl 1): S49-S60, 2007.

3. Halappa VG, Bonekamp S and Corona-Villalobos CP, et al: Intrahepatic cholangiocarcinoma treated with local-regional therapy: quantitative volumetric apparent diffusion coefficient maps for assessment of tumor response. Radiology 264: 285-294, 2012.

4. Poggi G, Amatu A and Montagna B, et al: OEM-TACE: a new therapeutic approach in unresectable intrahepatic cholangiocarcinoma. Cardiovasc Intervent Radiol 32: 1187-1192, 2009. 
5. Tan JC, Coburn NG, Baxter NN, Kiss A and Law CH: Surgical management of intrahepatic cholangiocarcinoma - a populationbased study. Ann Surg Oncol 15: 600-608, 2008.

6 . Thongprasert S: The role of chemotherapy in cholangiocarcinoma. Ann Oncol 16 (Suppl 2): i93-i96, 2005.

7. Liapi E and Geschwind JF: Chemoembolization for primary and metastatic liver cancer. Cancer J 16: 156-162, 2010.

8. Dumont AG, Yang Y, Reynoso D, Katz D, Trent JC and Hughes DP: Anti-tumor effects of the Notch pathway in gastrointestinal stromal tumors. Carcinogenesis 33: 1674-1683, 2012.

9. Brennan K and Clarke RB: Combining Notch inhibition with current therapies for breast cancer treatment. Ther Adv Med Oncol 5: 17-24, 2013.

10. Jonusiene V, Sasnauskiene A and Lachej N, et al: Down-regulated expression of Notch signaling molecules in human endometrial cancer. Med Oncol 30: 438, 2013.

11. Zhu H, Zhou X, Redfield S, Lewin J and Miele L: Elevated Jagged-1 and Notch-1 expression in high grade and metastatic prostate cancers. Am J Transl Res 5: 368-378, 2013.

12. Yu S, Zhang R, Liu F, Wang H, Wu J and Wang Y: Notch inhibition suppresses nasopharyngeal carcinoma by depleting cancer stem-like side population cells. Oncol Rep 28: 561-566, 2012.

13. Villanueva A, Alsinet $C$ and Yanger $K$, et al: Notch signaling is activated in human hepatocellular carcinoma and induces tumor formation in mice. Gastroenterology 143: 1660-1669, 2012.

14. Yabuuchi S, Pai SG and Campbell NR, et al: Notch signaling pathway targeted therapy suppresses tumor progression and metastatic spread in pancreatic cancer. Cancer Lett 335: 41-51, 2013.

15. Ye QF, Zhang YC, Peng XQ, Long Z, Ming YZ and He LY: siRNA-mediated silencing of Notch-1 enhances docetaxel induced mitotic arrest and apoptosis in prostate cancer cells. Asian Pac J Cancer Prev 13: 2485-2489, 2012.
16. Yoon HA, Noh MH and Kim BG, et al: Clinicopathological significance of altered Notch signaling in extrahepatic cholangiocarcinoma and gallbladder carcinoma. World J Gastroenterol 17: 4023-4030, 2011.

17. Munoz M, Henderson M, Haber M and Norris M: Role of the MRP1/ABCC1 multidrug transporter protein in cancer. IUBMB Life 59: 752-757, 2007.

18. Baguley BC: Multidrug resistance in cancer. Methods Mol Biol 596: 1-14, 2010.

19. Shao H, Huang Q and Liu ZJ: Targeting Notch signaling for cancer therapeutic intervention. Adv Pharmacol 65: 191-234, 2012.

20. Wang M, Xue L and Cao Q, et al: Expression of Notch1, Jagged1 and $\beta$-catenin and their clinicopathological significance in hepatocellular carcinoma. Neoplasma 56: 533-541, 2009.

21. Trehanpati N, Shrivastav S and Shivakumar B, et al: Analysis of Notch and TGF- $\beta$ signaling expression in different stages of disease progression during hepatitis B virus infection. Clin Transl Gastroenterol 3: e23, 2012.

22. Pei J, Tang Z, Zang G and Yu Y: Blockage of Notch1 signaling modulates the T-helper (Th)1/Th2 cell balance in chronic hepatitis B patients. Hepatol Res 40: 799-805, 2010.

23. Nefedova Y, Cheng P, Alsina M, Dalton WS and Gabrilovich DI Involvement of Notch-1 signaling in bone marrow stromamediated de novo drug resistance of myeloma and other malignant lymphoid cell lines. Blood 103: 3503-3510, 2004.

24. Zou J, Li P and Lu F, et al: Notch1 is required for hypoxiainduced proliferation, invasion and chemoresistance of T-cell acute lymphoblastic leukemia cells. J Hematol Oncol 6: 3, 2013. 